\title{
Promoção da Saúde na Atenção Básica: estudo baseado no método de Paulo Freire
}

\author{
Health promotion in primary care: \\ study based on the Paulo Freire method
}

Ivonete Teresinha Schülter Buss Heidemann ${ }^{1}$

Antonio de Miranda Wosny ${ }^{1}$

Astrid Eggert Boehs ${ }^{1}$

${ }^{1}$ Departamento de Enfermagem, Centro de Ciências da Saúde, Universidade Federal de Santa Catarina. R. Deputado Antônio Edu Vieira até 999/1000, Pantanal. 88.040-000 Florianópolis SC Brasil. ivonete.heidemann@ufsc.br
Abstract The scope of this study is to analyze the implementation of health promotion actions in the working process of the Family Health Teams of a city in the state of Santa Catarina. It involves research adopting a qualitative approach linked to the methodological benchmark of Paulo Freire, consisting of three dialectic moments: thematic investigation; encoding and decoding; critical revelation. Fifteen Culture Circles were conducted, covering five district health units, with the participation of 70 professionals. Each meeting was scheduled to last two hours with an average attendance of thirteen participants of the Family Health teams. The research revealed that there are limitations to the implementation of health promotion as a key element of participatory action together with the community. It also highlighted the importance of interdisciplinarity and intersectorality between workers and the city, state and federal manager. The commitment to the principles of the Unified Health System (SUS) and health promotion also presents itself as a challenge to improve the quality of life of the population.

Key words Nursing. Health promotion. Family health
Resumo Analisa a incorporação das ações de Promoção da Saúde no processo de trabalho das Equipes de Saúde da Família de um município catarinense. Pesquisa de abordagem qualitativa articulada com o referencial metodológico de Paulo Freire, consistindo de três momentos dialéticos: Investigação temática; Codificação e decodificação; Desvelamento crítico. Foram realizados quinze Círculos de Cultura, abrangendo cinco unidades distritais de saúde, com a participação de 70 profissionais. Cada encontro previa duas horas de duração e teve a média de treze participantes das equipes de Saúde da Família. A pesquisa desvelou que existem limitações à incorporação da promoção da saúde como elemento fundamental da prática participativa com a comunidade. Destacou também a importância da interdisciplinaridade e intersetorialidade articulada entre trabalhador e gestor municipal, estadual e federal. O comprometimento com os princípios do SUS e da promoção da saúde apresenta-se, ainda, como desafio para a melhoria da qualidade de vida da população.

Palavras-chave Enfermagem, Promoção da saúde, Saúde da família 


\section{Introdução}

A promoção da saúde permanece em constate debate teórico e conceitual e apresenta-se, ainda multifacetada, nos diversos campos da saúde humana e sociedade. Entretanto, a concepção resultante da Carta de Ottawa, de 1986, permanece como principal ideário norteador das políticas governamentais para a área da saúde. Assim sendo, a definição que prevalece $^{1}$ é aquela que considera a Promoção da Saúde como o processo de capacitação de indivíduos, famílias e comunidades para aumentar o controle sobre os determinantes de saúde e atuar na melhoria de sua qualidade de vida e saúde. A promoção de saúde vai para além de um estilo de vida saudável e extrapola o setor, para um bem-estar global, transformando a ideia, vigente até a década de 70, de uma saúde focada nos determinantes biológicos e centralizada nas preocupações com a doença de indivíduos e populações².

No final do Séc. XX ocorreram mudanças no paradigma biomédico, impulsionando o movimento da Promoção da Saúde a nível mundial, que influenciaram a Reforma Sanitária brasileira, a qual culminou com a criação do Sistema Único da Saúde (SUS), em 1990. Com a criação da ESF, o governo aprova em 2006, através da Portaria no 648 a Política Nacional de Atenção Básica ${ }^{3}$ (PNAB), caracterizando Atenção Básica como um conjunto de ações de saúde, no âmbito individual e coletivo, que abrangem a promoção e a proteção da saúde, a prevenção de agravos, o diagnóstico, o tratamento, a reabilitação e a manutenção da saúde. Em 2006, a Política Nacional de Promoção da Saúde (PNPS) reafirma as estratégias da Carta de Ottawa. Esta política adota ações públicas que superem a ideia de cura e reabilitação implantando as diretrizes e ações para Promoção da Saúde em consonância com os princípios do SUS, como o Pacto pela Saúde, pela Vida e em Defesa do SUS e sua Gestão e em todas esferas de governo ${ }^{4}$.

Em 2012, o Ministério da Saúde reafirma a Política Nacional de Atenção Básica (PNAB), atualizando conceitos na política e introduzindo novos elementos referentes à Atenção Básica na ordenação das Redes de Atenção ${ }^{3}$.

A promoção da saúde reforça o conceito de determinação social da saúde, com objetivo de impactar favoravelmente a qualidade de vida. Ações intersetoriais e intrassetoriais são imprescindíveis para ampliação da consciência sanitária, com direitos e deveres da cidadania, educação em saúde para mudança das condições de vida da população ${ }^{5}$. Neste sentido, as estratégias da promoção da saúde são as que possibilitam desenvolver a autonomia e fortalecer processos políticos e sociais que permitam a transformação dos determinantes estruturais que reafirmam os princípios do SUS. A Promoção da Saúde consolida-se na Estratégia da Saúde da Família reforçando os princípios do SUS, especialmente o da integralidade na atenção à saúde e o da participação social ${ }^{6}$.

Assim, este estudo relata resultados de investigação com objetivo de identificar e analisar a compreensão conceitual da Promoção da Saúde, assim como a incorporação das estratégias no processo de trabalho das equipes de Saúde da Família do município de Florianópolis. Neste município, a Saúde da Família deu-se a partir de 1996 e atualmente há aproximadamente 115 equipes, o que corresponde a 433.158 da população. Apresenta uma ampla rede pública de prestação de serviços em Atenção Básica de Saúde, com 48 Unidades de Saúde e 5 Distritos Sanitários distribuídas nas regiões norte, leste, sul, oeste, centro e continente ${ }^{7}$.

\section{Metodologia}

A opção pelo Itinerário de Pesquisa de Paulo Freire justifica-se pela tradição de Investigação de abordagem qualitativa do Núcleo de Pesquisa e Extensão em Promoção da Saúde e Enfermagem (NEPEPS) do Programa de Pós Graduação em Enfermagem da Universidade Federal de Santa Catarina. No Brasil, o referencial de Paulo Freire solidifica-se na área educacional, entretanto, a prática de pesquisa em saúde apresenta-se em expansão, embora lenta, considerando que em muitos países, as ações de promoção da saúde estão consolidadas e utilizam este referencial como opção metodológica importante.

Neste estudo, o método Paulo Freire apresentou-se oportuno, considerando que para a investigação dos temas, prevê uma relação de diálogo do pesquisador com os sujeitos da pesquisa. Desvela a realidade social, o que está oculto, permitindo que as reflexões dos participantes os levem a novas propostas de ação sobre o cotidiano de promoção da saúde.

A investigação ocorreu em três momentos dialéticos e interdependentes, de acordo com Freire $^{8}$, em Círculos de Cultura, acatando as etapas tradicionais do método: Investigação dos Temas Geradores; Codificação e Descodificação; Desvelamento Crítico. O Círculo de Cultura é um termo criado por Freire, representado por um espaço dinâmico de aprendizagem e troca de conhecimentos. Os sujeitos se reúnem no processo de educação para investigar temas de interesse do 
próprio grupo. Representa uma situação-problema de situações reais, que leva à reflexão da própria realidade, para, na sequencia, decodificá-la e reconhece-la ${ }^{8,9}$. Além disso, o Esquema do Itinerário de Pesquisa adaptado de Saupe ${ }^{10}$ detalha esses passos, conforme Figura 1.

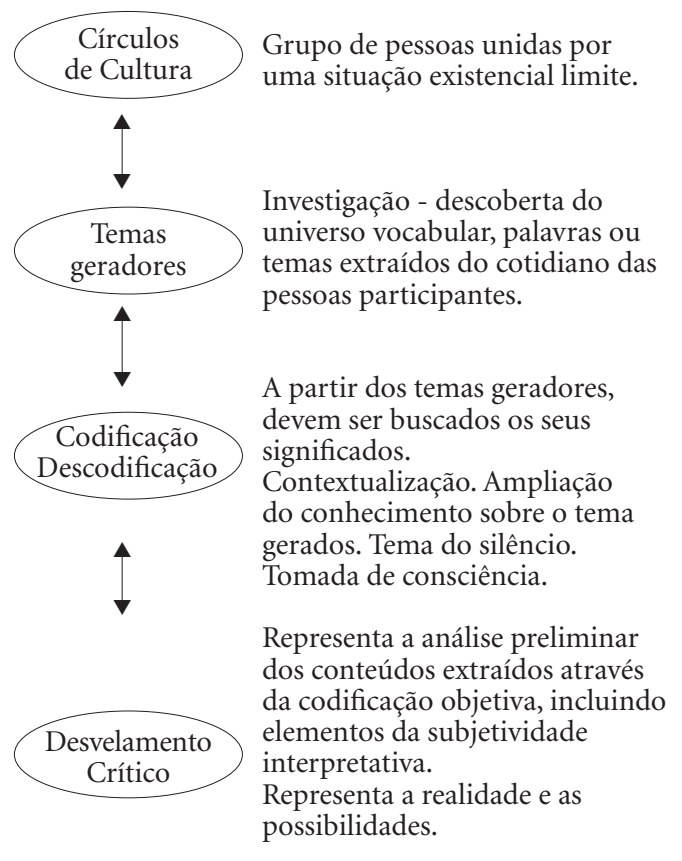

Figura 1. Esquema do Itinerário de Pesquisa de Paulo Freire.

Fonte: adaptado de Saupe ${ }^{10}$.
Para alcançar os objetivos da pesquisa foram organizados três (03) Círculos de Cultura em cada uma das cinco Unidades de Saúde envolvidas no estudo. Estas Unidades são integrantes dos Distritos de Saúde da área Central, Norte, Sul, Leste e Continental. Os Círculos de Cultura foram realizados com duração de duas horas e tiveram a participação total de cinquenta e seis (56) pesquisadores, profissionais, integrantes das equipes de Saúde da Família, acadêmicos de enfermagem: dez (10) pesquisadores, dois (2) Médicos, seis (6) Enfermeiros, quatro (4) Odontólogos, sete (7) Técnicos de Enfermagem e vinte e dois (22) Agentes Comunitários de Saúde e quatro (4) Acadêmicos de Enfermagem (Tabela 1).

A escolha das equipes de ESF foi por sorteio, considerando que na maioria delas havia mais de uma equipe. Os pesquisadores envolvidos tiverem capacitação prévia para apreender sobre o Método do Círculo de Cultura e aplicá-lo com os sujeitos da pesquisa. A entrada no campo foi pactuada na reunião mensal das Unidades envolvidas no estudo.

Nesta reunião os pesquisadores se apresentaram, detalharam os objetivos da pesquisa e agendaram o primeiro círculo para a reunião semanal da equipe de ESF.

Os Círculos de Cultura foram desenvolvidos através de dinâmica própria do método, objetivando conhecer inicialmente a percepção dos profissionais sobre promoção da saúde. Posteriormente, o debate encaminhou-se para identificação das situações que facilitavam e dificultavam a inserção das estratégias de promoção da saúde no processo de trabalho das equipes e suas respectivas áreas de atuação. Estas situações configuram-se os temas a serem investigados.

Tabela 1. Distribuição dos profissionais de saúde por US sorteada de Florianópolis.

\begin{tabular}{lccccc}
\hline \multirow{2}{*}{ Categoria profissional } & \multicolumn{5}{c}{ Local } \\
\cline { 2 - 6 } & $\begin{array}{r}\text { Unidade de } \\
\text { Saúde 1 }\end{array}$ & $\begin{array}{c}\text { Unidade de } \\
\text { Saúde 2 }\end{array}$ & $\begin{array}{c}\text { Unidade de } \\
\text { Saúde 3 }\end{array}$ & $\begin{array}{c}\text { Unidade de } \\
\text { Saúde 4 }\end{array}$ & $\begin{array}{c}\text { Unidade de } \\
\text { Saúde 5 }\end{array}$ \\
\hline Enfermeiro & 1 & 1 & 2 & 1 & 1 \\
Médico & - & - & - & 1 & 1 \\
Odontólogo & 1 & 1 & 1 & - & 1 \\
Técnico de enfermagem & 2 & 1 & 1 & 2 & 1 \\
ACS & 5 & 3 & 4 & 5 & 5 \\
Auxiliar de Consultório Dentário & - & - & 1 & - & - \\
Acadêmicos de enfermagem & - & - & & 4 & - \\
Pesquisadores & 2 & 2 & 2 & 2 & 2 \\
TOTAL & 11 & 8 & 11 & 15 & 11 \\
\hline
\end{tabular}

Fonte: Círculos de Cultura. 
A investigação dos temas ocorreu em cada Unidade de Saúde sob a mediação de um (1) pesquisador e um (1) colaborador. Os temas investigados, codificados e desvelados foram registrados pelos participantes em tarjetas e painéis que destacavam palavras-chaves, em cores diferentes, significando os limites e as potencialidades da promoção da saúde. Além disso, os pesquisadores registravam em diário de campo detalhes importantes das manifestações dos sujeitos, assim como da organização e desenvolvimento do Círculo de Cultura.

A análise dos dados ocorreu com a participação de todos os integrantes dos Círculos de Cultura, através da leitura cuidadosa, reflexão e interpretação dos temas destacados. Elaboraram-se quadros sintéticos (dados produzidos) destacando as atividades realizadas pela equipe de saúde, interpretadas como promoção da saúde. Posteriormente, os participantes dos Círculos de Cultura, descodificaram o significado dos temas produzindo um novo olhar sobre a Promoção da Saúde.

A pesquisa atendeu os requisitos da Resolução 196/1996 ${ }^{11}$, do Ministério da Saúde e foi aprovada pelo Comitê de Ética da Universidade Federal de Santa Catarina (UFSC). O Termo de Consentimento Livre e Esclarecido (TCLE) foi assinado por vinte e cinco (25) pessoas que participaram da pesquisa. Para garantir o anonimato dos mesmos os profissionais tanto de nível superior como os de nível médio foram identificados como profissionais, independentemente de categoria (médicos, enfermeiros, odontológos e técnicos), os ACS permaneceram como agentes comunitários de saúde e os locais da pesquisa foram identificados por número. A assinatura do termo de consentimento foi solicitada aos participantes e a participação na pesquisa foi voluntária.

\section{Resultados e discussão}

Os primeiros Círculos de Cultura realizados nas Unidades de Saúde da Família de Florianópolis tiveram o objetivo de apresentar a pesquisa aos profissionais, destacando seus objetivos e metodologia.

A primeira temática em debate relacionava-se com as atividades desenvolvidas, facilidades e dificuldades que as equipes percebiam para promover a saúde na sua área de abrangência. O objetivo era identificar e refletir se essas ações incorporavam as estratégias da Carta de Ottawa no processo de trabalho desenvolvido pelas equipes. A identificação dos temas foi realizada através de exposição escrita em papel pardo e tarjetas, onde cada participante manifestava seu entendimento de promoção da saúde, destacando alguma dificuldade de expressão. Foram estimulados a expressar as práticas que entendiam como promoção da saúde. Entretanto, percebeu-se dificuldade de entender claramente estas atividades de promoção e sua delimitação conceitual de acordo com os princípios da Carta de Ottawa. Evidenciaram-se limitações de uma política de promoção da saúde, manifestado na prática dos profissionais através da incapacidade de reconhecer diferenças entre o termo prevenção de doença e promoção da saúde, mesmo assim expressam alguma compreensão do tema, mesmo ingenuamente: "a promoção vai muito além da Unidade de Saúde" (profissional). Os temas geradores que emergiram dos Círculos de Cultura, e que representam as atividades de promoção, facilidades e dificuldades, que foram desenvolvidos no primeiro Círculo de Cultura foram: Atendimento Individual, Imunização, Atendimento Coletivo, Acolhimento, Ações Comunitárias, Visita Domiciliar, Violência, Intersetorialidade, Relacionamento da Equipe de Saúde, Atenção ao Meio Ambiente, Dificuldades de Recursos para o Lazer, Saúde do Escolar, Organização do Trabalho Deficiente, Estrutura Material Precária, Dificuldade em Criar Vínculo, Programas Assistenciais, Referência e Contra Referência, Recursos operacionais.

Através da Investigação Temática, independente da categoria profissional, os participantes revelaram sua compreensão das atividades da promoção da saúde. Relacionaram procedimentos de rotina, ações comunitárias, atividades de educação em saúde, programas e projetos assistenciais governamentais ou não como exemplo de ações de promoção da saúde. Com isso, identificou-se que mesmo ocorrendo ações de promoção da saúde, elas não são entendidas como política e objetivos que devem ser incorporadas pelas equipes no seu processo de trabalho. Em pesquisa de revisão integrativa realizada ${ }^{2}$ sobre concepções de promoção da saúde a temática relacionada a esse assunto, destaca ainda, uma compreensão ingênua, tradicional persistindo o enfoque na mudança de estilos de vida, para alcançar um estado ótimo de saúde. A promoção da saúde é destacada como forma de ajudar a manter a saúde de aposentados, beneficiar indivíduos e comunidades, melhorar o comportamento de pessoas com deficiência, auxiliá-las em situações de doenças crônicas, apoiar a saúde de idosos, jovens e adultos e de pessoas pobres para obter maiores expectativas de vida ${ }^{12}$.

Percebe-se carência de capacitação profissional para entendimento e incorporação da Política Nacional de Promoção da Saúde, assim como das Diretrizes Operacionais da Estratégia de Saúde da Família. 
Em relação às facilidades para desenvolver as práticas rotineiras da Unidade, interpretadas como de promoção da saúde, os participantes destacaram quatro (4) temas: 1) Funcionamento da Unidade e Equipes de ESF; 2) proximidade da Unidade Básica de Saúde; 3 ) Recursos Operacionais e 4) Processo de Trabalho.

Com relação ao funcionamento da Unidade, apontaram que reuniões das equipes, horário estendido e participação nos grupos de educação em saúde, promovem o desenvolvimento das praticas consideradas como promoção da saúde. Além disso, enfatizam que recursos materiais adequados contribuem para o bom funcionamento da Unidade. Percebe-se que as ações de saúde desenvolvida pelos profissionais aos usuários dependem da dinâmica da Unidade, visto que é necessário reestruturar a conduta dos profissionais inseridos na Estratégia Saúde da Família, uma vez que têm como função a promoção da saúde, em uma lógica intersetorial e interdisciplinar ${ }^{13}$.

Como segunda temática aponta a proximidade da Unidade de Saúde com a população, possibilitando a operacionalização de intervenções para uma perspectiva mais ampliada de promoção da saúde. Esta aproximação promove o vínculo, a acessibilidade e o desenvolvimento de um ambiente favorável da saúde de acordo com os princípios do SUS. O estabelecimento de vínculo, principalmente através das visitas domiciliares, atividades educativas, promove a relação mais próxima entre a comunidade e a equipe de saúde, e facilita a adesão da população ao serviço ${ }^{14}$.

No tema operacionalização de recursos, os participantes revelaram que o desenvolvimento das atividades de saúde depende de infraestrutura adequada na Unidade. Com recursos apropriados, providenciam-se boa divulgação e operacionalização das atividades, inclusive as de promoção da saúde. Os recursos financeiros são fundamentais para o desenvolvimento das ações de promoção da saúde. Material didático e de divulgação, assim como espaço físico adequado favorecem o interesse da comunidade para participação nas atividades da Unidade Básica de Saúde.

A temática do processo de trabalho, dialogada nos Círculos de Cultura desvelou que equipes responsáveis e atuantes promovem o fortalecimento do vínculo e acesso da comunidade na Unidade de Saúde. Este fato revela a importância da ESF na implantação e desenvolvimento das ações de promoção da saúde. A abordagem interdisciplinar permite o desenvolvimento de atividades de promoção da saúde, com continuidade e acompanhamento, maior envolvimento com os familiares e comunidade. É relevante que os membros da equipe de saúde da família atuem em harmonia interagindo com a comunidade, com amor e paciência, vontade conjunta do grupo A união faz a força (profissional).

Por outro lado verifica-se, ainda que uma das grandes dificuldades enfrentadas pelo ESF é o processo de trabalho médico-centrado que opera na produção do cuidado e da cura. Em pesquisa realizada sobre representações sociais dos trabalhadores sobre o PFS no Distrito Federal ${ }^{15}$, identificou-se que os trabalhadores expressaram que o processo de trabalho é bastante complexo, requer divisão de tarefas, porque envolve a execução de diversas atividades. Isto por sua vez exige sofisticada síntese de diferentes saberes e articulação das ações de diversos níveis de complexidade.

Observa-se pela literatura que o trabalho em equipe é a base para ações integral na saúde e voltada à família e seu contexto. A Estratégia Saúde da Família tem a responsabilidade de viabilizar o SUS como um modelo assistencial ampliado, cuja ênfase estaria na promoção da saúde, com o objetivo de alterar a volumosa demanda espontânea por ações e serviços de saúde, por meio da organização e do controle da mesma ${ }^{16}$.

As dificuldades para desenvolver as atividades de promoção da saúde, foram apontadas em três dimensões: Limitação de recursos humanos e materiais; educação permanente das equipes para o processo de trabalho na ESF; dificuldades para participação e vínculo da população com a Unidade de Saúde; e, modelo hegemônico, centrado na consulta médica.

O diálogo no Círculo de Cultura revelou que a limitação dos recursos humanos está relacionada à demanda maior de necessidades de saúde em relação à oferta de serviços. O número de equipes de Saúde da Família nas unidades pesquisadas, ainda não corresponde ao preconizado como ideal da Política Nacional de Atenção Básica e ESF. Outra demanda percebida refere-se à agilização para atendimento de necessidades de saúde de maior complexidade. De acordo com os participantes do estudo este fato gera sentimento de impotência para quem cuida da população. Além disso, relataram a insuficiência de recursos materiais para o desenvolvimento de ações diretas com a comunidade, especialmente àquelas relacionadas à locomoção das equipes para atender necessidades de atenção e promoção da saúde distantes da Unidade de Saúde.

Destacou-se situação crítica no processo de trabalho para executar as práticas de promoção da saúde. Percebe-se que há necessidade de constru- 
ção de conhecimento específico das bases teóricas da promoção da saúde, assim como oportunidades reduzidas de educação permanente em metodologias ativas e criativas para abordagem coletivas com a comunidade.

O modelo tradicional de promoção da saúde mantém o foco na "culpabilização das vitimas"17, reforçando que os usuários são responsáveis pelos seus estilos de vida e comportamentos não saudáveis. Lamentam que a população reage reivindicando direitos para obter atendimento de saúde qualificado aludindo que "os usuários reclamam quando não recebem assistência rápida, são intolerantes e imediatistas" (profissional de saúde). Desse modo, destaca-se a dificuldade de articulação dos profissionais com a comunidade, responsabilizando-a pela desmobilização, não percebendo a sua corresponsabilidade neste processo. Entretanto, estes mesmos profissionais percebem a importância da organização comunitária e dos conselhos locais de saúde, mas transferem esta responsabilidade aos gestores caracterizando como falta de vontade política e organização do sistema de saúde. Aprofundando a investigação dos temas acima destacados, identifica-se que as equipes apresentam carência da educação continuada nas questões organizacionais dos serviços, com ênfase no processo comunicativo solidário, assim como formação com foco em metodologias participativas para trabalhos de promoção da saúde e cidadania.

Destaca-se ainda o que muitos autores ${ }^{18}$ já vêm apontando: que o fornecimento de intervenção de promoção da saúde com base na comunidade, nas famílias e indivíduos pode ajudar a reduzir tanto o peso da doença como suas despesas de saúde, melhorando a qualidade de vida. As pessoas que aderiram às atividades de promoção da saúde relataram significativamente menos adoecimento e menos despesas de saúde.

A partir da codificação, descodificação e desvelamento crítico das temáticas investigadas, foi apontando para a necessidade de maior dialogo sobre o conceito de promoção da saúde e prevenção da doença. As reflexões instigaram sobre a prática das equipes de Saúde da Família relacionando teoria e prática da promoção da saúde. Percebeu-se a necessidade de diferenciar claramente prevenção de promoção, considerando o entendimento ingênuo generalizado dos profissionais. Suas manifestações nos Círculos de Cultura apontam para o sujeito o qual "deve" evitar as doenças, e ser educada em saúde, isentando a responsabilidade do profissional da saúde, e responsabilizando o indivíduo, a sociedade e o Estado.
A promoção da saúde foi confundida na maioria das vezes com prevenção de doenças. Esta dificuldade de compreensão já foi evidenciada em uma pesquisa realizada sobre a Estratégia Saúde da Família (ESF) ${ }^{19}$ e análise da realidade social em município de SC. Neste trabalho, a maioria da equipe percebe a promoção da saúde no seu sentido coletivo e social, próximo da ação educativa e preventiva, mas com ênfase na transmissão de conhecimentos e confusão entre promoção e prevenção, o que reduz os desafios, as missões e as propostas da promoção da saúde na ESF.

\section{Considerações finais}

Retomando o objetivo do presente estudo que foi identificar e analisar a compreensão conceitual da Promoção da Saúde, assim como a incorporação das estratégias no processo de trabalho de equipes de Saúde da Família é possível afirmar que os resultados contribuíram para reflexão desta temática, mas destaca ainda a necessidade de aprofundamento na prática e formação dos profissionais da saúde.

A partir da investigação realizada nas Unidades de Saúde percebeu-se a necessidade de dialogar sobre o conceito de promoção e prevenção com as equipes de Saúde da Família. Instigaram-se reflexões sobre o que é promoção e como a mesma é inserida no cotidiano dos profissionais da saúde. Muitos não sabiam diferenciar prevenção de promoção, outros compreendiam que ambas eram sinônimos, exemplificando como ações realizadas nas Unidades.

Destaca-se, ainda que promoção da saúde esta relacionado com as pessoas, é um conceito amplo que para ser inserido na prática necessita do envolvimento intersetorial. Já a prevenção tem a ver com a ocorrência da doença, é um conceito mais centrado o qual antecipa uma ação para evitar que algo funcione e reduzir o risco de adoecer. Concomitante ao promovermos a saúde devemos prevenir a doença.

Recomendam-se novas pesquisas sobre as concepções de promoção de saúde e qualidade de vida dos profissionais da saúde que atuam tanto na assistência primária, secundária como terciária. Realizar ainda, pesquisas para conhecer estas concepções de outros profissionais como gestores, administradores das cidades e do campo, possíveis interlocutores dos profissionais de saúde para o exercício da intersetorialidade. 


\section{Colaboradores}

ITSB Heidemann, AM Wosny e AE Boehs trabalharam na concepção, metodologia e redação final do trabalho.

\section{Referências}

1. World Health Organization (WHO). The Ottawa charter for health promotion. Ottawa: WHO; 1986.

2. Heidemann ITSB, Boehs AE, Fernandes GCM, Wosny AM, Marchi JG. Promoção da saúde e qualidade de vida: concepções da carta de Ottawa em produção científica. Cienc Cuid Saude 2012 ; 11(3):613-619.

3. Brasil. Ministério da Saúde (MS). Secretaria de Atenção à Saúde. Departamento de Atenção Básica. Política Nacional de Atenção Básica. Brasília: MS; 2012.

4. Brasil. Ministério da Saúde (MS). Política Nacional de Promoção da Saúde. Brasília: MS; 2006.

5. Buss PM. O conceito de promoção da saúde e os determinantes sociais. Eco cidadania \& Meio Ambiente. [periódico na Internet]. 2010 fevereiro. [acessado 2013 jun 30]. [cerca de 15 p.]. Disponível em: http://www.ecodebate. com.br/2010/02/12/o-conceito-de-promocao-da-saude -e-os- determinantes-sociais-artigo-de-paulo-m-buss/

6. Durand M, Heidemann ITSB. Promoção da autonomia da mulher na consulta de enfermagem em saúde da família. Rev Esc Enferm 2013; 47(2):288-295.

7. Maceno PR. Desvelando as Ações de Promoção da Saúde das enfermeiras nos Grupos de Atendimentos Coletivos de Atenção Básica [dissertação]. Florianópolis: Universidade Federal de Santa Catarina; 2013.

8. Freire P. Pedagogia do oprimido. $42^{\mathrm{a}}$ ed. Rio de Janeiro: Paz e Terra; 2005.

9. Heidemann ITSB, Boehs AE, Wosny AM, Stulp KP. Incorporação teórico-conceitual e metodológica da educador Paulo Freire na pesquisa. Rev Bras Enferm 2010; 63(3):416-420.

10. Saupe R. Esquema do Itinerário de Pesquisa de Paulo Freire. [periódico na Internet]. 1999 [acessado 2013 jun 25]. [cerca de 10p.]. Disponível em: http://www.ccs.ufsc. br/enfermagem/educaçação.

11. Brasil. Ministério da Saúde (MS). Conselho Nacional de Saúde. Resolução nº 196 de 10 de outubro de 1996. Diretrizes e Normas Regulamentadoras de Pesquisas Envolvendo Seres Humanos. Diário Oficial da União 1996; 16 out.
12. Wilson DM, Palha P. A Systematic Review of Published Research Articles on Health Promotion at Retirement. Journal Nursing Scholarship 2007; 39(4):330-337.

13. Taddeo PS, Kilma WLG, AC, Annatália MAG, Giselle CO, Thereza MM. Acesso, prática educativa e empoderamento de pacientes com doenças crônicas. Cien Saude Colet 2012; 17(11): 2923-2930.

14. Oliveira EM, Spiri WC. Programa Saúde da Família: a experiência de equipe multiprofissional. Rev Saude Publica 2006; 40(4):727-733.

15. Shimizu HE, Reis LS. As representações sociais dos trabalhadores sobre o Programa Saúde da Família. Cien Saude Colet 2011; 16(8):3461-3468.

16. Uchimura KY, Bosi MLM. Habilidades e competências entre trabalhadores da Estratégia Saúde da Família. Interface (Botucatu) 2012; 16(40):149-160.

17. Carvalho SR. As contradições da promoção à saúde em relação à produção de sujeitos e a mudança social. Cien Saude Colet 2004; 9(3):669-678.

18. Rana AK, Wahlin A, Lundborg CS, Kabir ZN. Impact of health education on health-elated quality of life among elderly persons: results from a community-based intervention study in rural Bangladesh. Health Promotion International 2009; 24(1):36-45

19. Charles DT, Adir VG, Carine V, Cleonete EA. Estratégia saúde da família e análise da realidade social: subsídios para políticas de promoção da saúde e educação permanente. Cien Saude Colet 2011; 16(11):4295-4306.

Artigo apresentado em 11/05/2013

Aprovado em 30/06/2013

Versão final apresentada em 11/07/2013 